\title{
THE INTERACTIONS OF NITROGEN MOLECULES ADSORBED ON GRAPHITE
}

\author{
W. A. STEELE (*) \\ School of Chemistry, The University, Bristol, England.
}

\begin{abstract}
Résumé. - Les succès obtenus lors du traitement théorique des interactions gaz rares-graphite et $\mathrm{N}_{2}-\mathrm{N}_{2}$ permettent d'établir un modèle d'interaction moléculaire de $\mathrm{N}_{2}$ avec le graphite. La forme non sphérique de cette molécule est introduite dans le calcul de l'énergie gaz-solide. De plus, l'interaction entre paires de molécules $\mathrm{N}_{2}$ adsorbées fait intervenir l'énergie quadripolaire et la non-sphéricité. Les énergies calculées sont en bon accord avec celles obtenues à partir des chaleurs d'adsorption expérimentales. On en déduit les valeurs des barrières de rotation et translation sur la surface. Ces valeurs permettent de caractériser les états de mouvement dans certaines des phases découvertes récemment dans ce système.
\end{abstract}

\begin{abstract}
A model for the molecular interaction of $\mathrm{N}_{2}$ with graphite is proposed which is derived from successful treatments of the energies of rare gases on graphite and of the $\mathrm{N}_{2}-\mathrm{N}_{2}$ interaction. The non-spherical shape of this molecule is explicitly taken into account in the gas-solid energy ; in addition, the interaction of a pair of adsorbed $\mathrm{N}_{2}$ molecules in quadrupolar energy as well as non-sphericity. Calculated energies are in good agreement with estimates obtained from the experimental heats of adsorption. Barriers to rotation and to translation across the surface are estimated and used to characterize the states of motion in some of the recently discovered ordered phases in this system.
\end{abstract}

1. Introduction. - Since the beginning of quantitative studies of physical adsorption, nitrogen has been by far the most popular choice of adsorbate. The standard determination of surface area is of course the BET $\mathrm{N}_{2}$ method, which requires a determination of the monolayer capacity using the BET theory and then a calculation of surface area using an estimated value for the molecular area of $\mathrm{N}_{2}$ in a dense layer. In view of this, it is surprising to find that theoretical studies of the interaction energies of nitrogen molecules at solid surface [1] are quite sparse and relatively crude as well. This hiatus is made more noticeable by the recent thermodynamic and neutron diffraction studies of nitrogen adsorbed on the basal planes of graphitic carbon $[2,3]$. A wealth of new phenomena was observed in this work, including two different ordered phases as well as a dense fluid (in two dimensions). The calculations reported in this paper are aimed at improving our knowledge of the position and orientation dependence of the energy of a single $\mathrm{N}_{2}$ molecule interacting with the basal plane of graphite, as well as of the mutual interaction of these adsorbed molecules.

$\left({ }^{*}\right)$ Unilever Visiting Professor: permanent address : Department of Chemistry, Pennsylvania State University, University Park, Pennsylvania 16802, U.S.A.
In part, the absence of calculations of $\mathrm{N}_{2}$-solid energies can be traced to the lack of an adequate model for the angle and distance dependence of the interaction between a pair of $\mathrm{N}_{2}$ molecules; this problem is further complicated by the necessity of specifying appropriate combining rules for the parameters of the potential which are needed to go from the $\mathrm{N}_{2}-\mathrm{N}_{2}$ interaction to the $\mathrm{N}_{2}-\mathrm{C}$ interaction. However, this is no longer a serious impediment to progress. In recent years, model potentials for $\mathrm{N}_{2}-\mathrm{N}_{2}$ interaction have been extensively tested against the experimental data for this substance [4-6], and it may be said with some confidence that a successful model for this potential is now available. To be specific, the energy is represented as a sum :

$$
\frac{u_{\mathrm{aa}}\left(r, \theta, \theta^{\prime}, \delta \phi\right)}{k}=4\left(\frac{\varepsilon}{k}\right) \sum_{i, j=1}^{2}\left\{\left(\frac{\sigma}{r_{i j}}\right)^{12}-\left(\frac{\sigma}{r_{i j}}\right)^{6}\right\}
$$

where the indices $i, j$ denote the two atomic centres in each molecule in the pair, $r$ denotes the centre-of-symmetry separation, $\theta, \quad \theta^{\prime}$ and $\phi, \phi^{\prime}\left(\delta \phi=\phi-\phi^{\prime}\right)$ denote the polar and azimuthal angles of the molecular axis relative to the centre-of-symmetry line. This representation of the effect of molecular shape upon the interaction 
potential is referred to as the diatomic model. The atom-atom distances can be calculated from

$$
\begin{aligned}
r_{i j}^{2} & =\left[r+(-1)^{j}\left(\frac{L}{2}\right)\left(Z+(-1)^{i} Z^{\prime}\right)\right]^{2}+ \\
& +\frac{L^{2}}{4}\left[X+(-1)^{i} X^{\prime}\right]^{2}+\frac{L^{2}}{4}\left[Y+(-1)^{i} Y^{\prime}\right]^{2}
\end{aligned}
$$

with $Z=\cos \theta, X=\sin \theta \cos \phi, Y=\sin \theta \sin \phi$.

In the case of $\mathrm{N}_{2}$, the best values for the parameters of the potential are : $\varepsilon / k=35 \mathrm{~K}$; $\sigma=3.32 \AA ; L=1.10 \AA=$ the $\mathrm{N}-\mathrm{N}$ bond length. In addition to this atom-atom energy, the quadrupolar interaction between these molecules is an important part of the total. This energy is accurately equal to that of a pair of ideal quadrupoles :

$$
\begin{aligned}
& \frac{u_{\mathrm{QQ}}\left(r, \theta, \theta^{\prime}, \delta \phi\right)}{k}= \\
& =\frac{3}{4} \frac{Q^{2}}{k r^{5}}\left\{1-5\left(Z^{2}+Z^{\prime 2}\right)+17\left(Z Z^{\prime}\right)^{2}+\right. \\
& \left.+2\left(X X^{\prime}+Y Y^{\prime}\right)^{2}-16 Z Z^{\prime}\left(X X^{\prime}+Y Y^{\prime}\right)\right\},
\end{aligned}
$$

where $Q$ is the molecular quadrupole moment. Using the known value of $Q[7]$ and $\sigma=3.32 \AA$, one finds that

$$
\frac{3 Q^{2}}{4 k \sigma^{5}}=30 \mathrm{~K}
$$

Over the past few years, either the model represented by eq. (1.1) and (1.3) or various minor modifications of it has been used extensively in computations of the properties of nitrogen; these include second virial coefficients [4]; computer simulations of dynamical and thermodynamic properties of the liquid [5]; and solid state properties [6], including crystal structure and energy as well as the phonon spectrum. The agreement between theory and experiment is good, and indicates that the size, shape and energy well depth of nitrogen molecules cannot be far from the values given by this model; the current uncertainties are estimated to be roughly $2 \%$ in size and shape and $5 \%$ in well depth.

In previous theoretical work, it has been established that the rare gas-graphite interactions are rather well approximated by a sum over the solid of the pair-wise atom-atom energies $[1,8]$. It is evident that this model can be readily extended to the $\mathrm{N}_{2}$-solid potential if one starts from the diatomic model for nitrogen. If the position of the centre of symmetry of the molecule relative to the surface lattice is denoted by $\mathbf{r}$ and its orientation by $\Omega$, one can write

$$
u_{\mathrm{s}}(\mathbf{r}, \Omega)=\sum_{i=1}^{2} \sum_{k} u_{\mathrm{as}}\left(r_{i k}\right)
$$

where $u_{\text {as }}\left(r_{s k}\right)$ is the interaction between atomic centre $i$ in the molecule and atom $k$ in the solid. It is assumed that $u_{\text {as }}\left(r_{i k}\right)$ has the (12-6) form of eq. (1.1) with parameters $\varepsilon_{\mathrm{gs}}, \sigma_{\mathrm{gs}}$; values for these parameters are obtained from the simple combining rules :

$$
\begin{aligned}
\varepsilon_{\mathrm{gs}} & =\sqrt{\varepsilon_{\mathrm{N}} \varepsilon_{\mathrm{C}}} \\
\sigma_{\mathrm{gs}} & =\left(\sigma_{\mathrm{N}}+\sigma_{\mathrm{C}}\right) / 2 .
\end{aligned}
$$

The C-C well depth and size parameter are reasonably well known from calculations of the rare gas-graphite energy [8]; one has $\varepsilon_{c} / k=28$ and $\sigma_{\mathrm{C}} / \boldsymbol{k}=3.40 \AA$. Although the geometric mean rule for the well depth is known to fail when the component well depths differ greatly [9], all combining rules work equally well when the differences are small; thus values of

$$
\begin{aligned}
\varepsilon_{\mathrm{N}-\mathrm{C}}^{\prime} / k & =31 \mathrm{~K} \\
\sigma_{\mathrm{N}-\mathrm{C}} & =3.36 \AA
\end{aligned}
$$

would be quite realistic.

One further assumption is needed before the calculation can proceed : namely, that the electric field gradient at the solid surface is small enough to allow one to neglect the quadrupole-field gradient contribution to the gas-solid energy. The results of such a calculation of the $\mathrm{N}_{2}$-graphite energy will be presented in the following section and it will be shown that the average energy compares well with the value obtained from the heat of adsorption at zero coverage. Subsequently, the interactions of pairs of adsorbed $\mathrm{N}_{2}$ molecules will be calculated and finally, speculations concerning the energetics of dense, ordered monolayers will be presented.

2. Gas-solid energy. - The net interaction of a linear molecule with a single crystal surface is a function of five variables, which we shall denote by: $z$, the perpendicular distance between the molecular centre of symmetry and the surface plane ; $\tau$, a two-dimensional vector parallel to the surface which specifies the position of the centre of symmetry relative to the surface lattice; $\beta$, the angle between the molecular symmetry axes and the normal to the surface ; and $\alpha$, the azimuthal angle of the symmetry axis (relative to the plane defined by the surface normal and a lattice cell vector). It will be convenient to specify $\tau$ by its length $\tau$ and orientation angle $\gamma$, relative to the lattice vector $\mathbf{a}_{1}$. The unit lattice cell for the graphite surface is shown in figure 1 , together with a nitrogen molecule at an arbitrary position over the lattice (but lying parallel to the surface).

The $\mathrm{N}_{2}$-solid energy is most readily calculated and represented by invoking the Fourier expansion of the gas-solid energy discussed elsewhere [10]. This approach is particularly useful for gas-graphite interactions because one can truncate the series after the first set of $\tau$-dependent terms without 


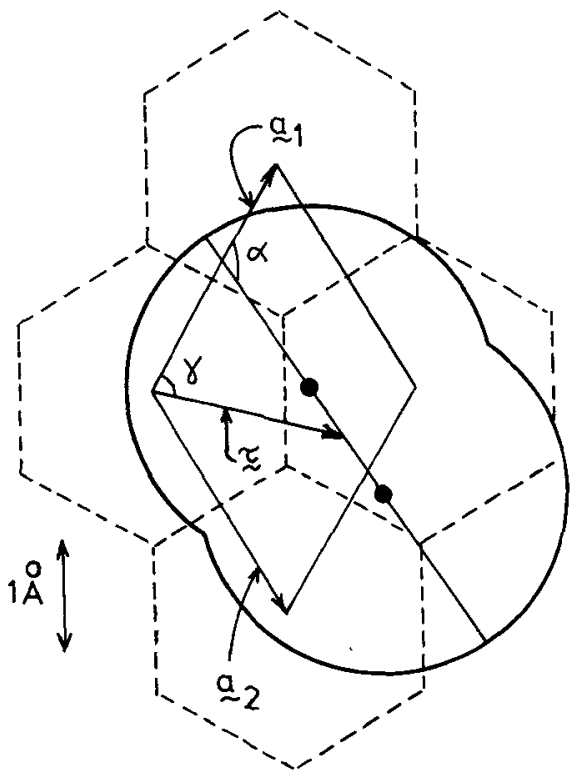

FIG. 1. - A schematic sketch of a nitrogen molecule lying flat on a graphite basal plane. The graphite lattice is shown by dashed lines ; the rhombic surface unit cell is defined by the vectors $a_{1}$, $\mathbf{a}_{2}$; nitrogen atoms are indicated by black dots and lie on the molecular symmetry axis ; $\gamma$ denotes the orientation of the centre-of-symmetry position vector relative to $\mathfrak{a}_{1}$, and $\alpha$ denotes the orientation of the symmetry axis. The fused circles outline the molecule (represented as a pair of atoms of diameter $3.32 \AA$ ).

appreciable loss of accuracy. For diatomic model, one has terms for each atom in the molecule interacting with the solid ; thus

$$
\begin{aligned}
& u_{s}(z, \tau, \beta, \alpha)=w_{0}\left(z+\frac{L}{2} \cos \beta\right)+ \\
& +w_{0}\left(z-\frac{L}{2} \cos \beta\right)+w_{1}\left(z+\frac{L}{2} \cos \beta\right) \sum_{n=1}^{3} \times \\
& \times \cos \left[g \tau \sin \left(\gamma+\frac{2 \pi n}{3}\right)+\right. \\
& \left.\quad+\frac{g L \sin \beta}{2} \sin \left(\alpha+\frac{2 \pi n}{3}\right)\right]+ \\
& \left.+w_{1}\left(z-\frac{L}{2} \cos \beta\right) \frac{\sum_{n=1}^{3} \times}{3}\right)- \\
& \times \cos \left[g \tau \sin \left(\gamma+\frac{2 \pi n}{3}\right)\right. \\
& \left.\quad-\frac{g L \sin \beta}{2} \sin \left(\alpha+\frac{2 \pi n}{3}\right)\right] .
\end{aligned}
$$

The length of the relevant reciprocal lattice vector $g$ is $2.95 \mathrm{~A}^{-1}$; thus, $g L / 2=1.62$. For a (12-6) atom-atom power law, the coefficients $w_{0}(z)$ and $w_{1}(z)$ are known to be given by $[10]$

$$
w_{0}(z)=\frac{4 \pi \varepsilon_{\mathrm{gs}}}{\left(a_{\mathrm{s}} / q\right)} \sum_{m=0}^{\infty}\left\{\frac{1}{5} \frac{\sigma_{\mathrm{gs}}^{12}}{(z+m d)^{10}}-\frac{1}{2} \frac{\sigma_{\mathrm{gs}}^{6}}{(z+m d)^{4}}\right\}
$$

where $a_{s}$ is the unit cell area and $d$ is the separation between atomic planes (parallel to the surface); for the graphite unit cell of figure 1 ,

$$
w_{1}(z)=\left(\frac{2 \pi \varepsilon_{\mathrm{gs}}}{a_{\mathrm{s}}}\right)\left\{\frac{\sigma_{\mathrm{gs}}^{12} g^{5}}{480 z^{5}} K_{5}(g z)-\frac{\sigma_{\mathrm{gs}}^{6} g^{2}}{z^{2}} K_{2}(g z)\right\}
$$

where $K_{n}$ is a modified Bessel function of the second kind. If we define reduced distances $z^{*}=z / a, \quad d^{*}=d / a, \quad$ where $a=$ cell edge length $=2.46 \AA$, substitution of numerical values into equations (2.2) and (2.3) gives

$$
\begin{array}{r}
\frac{w_{0}\left(z_{ \pm}\right)}{k}=900 \sum_{l=1,2} \sum_{m=0}^{\infty}\left\{\frac{8.431}{\left(z_{ \pm}^{*}+1.382 m\right)^{10}}-\right. \\
\left.-\frac{3.246}{\left(z_{ \pm}^{*}+1.382 m\right)^{4}}\right\}
\end{array}
$$

where $z_{ \pm}^{*}$ is the atomic position in reduced units and equals $z^{*}+0.224(-1)^{t} \cos \beta$; and

$$
\begin{aligned}
\frac{w_{i}(z)}{k}=225 \sum_{l=1.2}\left\{\frac{1771}{z_{. \pm}^{* 5}} K_{s}\left(7.26 z_{ \pm}^{*}\right)-\right. \\
\left.\quad-\frac{342.2}{z_{ \pm}^{* 2}} K_{2}\left(7.26 z_{ \pm}^{*}\right)\right\} .
\end{aligned}
$$

Of course, $w_{0}(z, \beta)$ is the energy of a $\mathrm{N}_{2}$ molecule averaged over all $\tau$; i.e., over the surface lattice at fixed distance and orientation relative to it.

We shall soon see that the modulation of this energy (given by the terms involving $w_{1}\left(z_{\exists}^{*}\right)$ ) is quite small for separation distances corresponding to the minimum in $w_{0}\left(z^{*}, \beta\right)$; thus, a calculation of the average energy is of interest in its own right as well as being a necessary step in the full computation. It is a simple matter to sum eq. (2.4) over the lattice planes and over the two atoms in the $\mathrm{N}_{2}$ molecule. Plots of the result are shown in figure 2 for various molecular orientations. Also shown is the energy

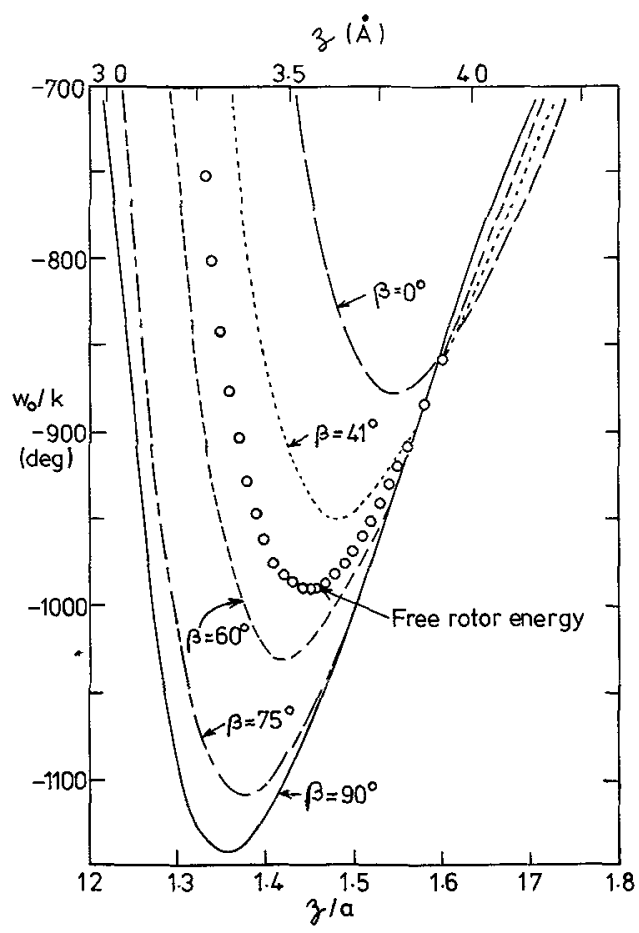

FIG. 2. - Position-averaged gas-solid interaction of a $\mathrm{N}_{2}$ molecule over a graphitic solid. The energy is plotted as a function of the centre-of-symmetry position above the surface plane for various values of the tilt angle $\beta$. Also shown is the energy obtained after averaging over $\beta$. 
averaged over orientation assuming that all values of $\beta$ are equally probable. It is evident that tumbling motion in this system is strongly coupled to motion perpendicular to the surface; that configurations with the molecule lying flat are the most probable, but at temperatures $\simeq 80 \mathrm{~K}$ the librational motion will be appreciable. These comments can be made more quantitative by expanding $w_{0} / k$ about its minimum :

$$
\frac{w_{0}}{k}=-1142^{\circ}+\frac{1}{2} k_{z}(\delta z)^{2}+\frac{1}{2} k_{\beta}(\delta \beta)^{2} .
$$

The force constants are :

$$
k_{z}=3540 \mathrm{deg} / \mathrm{A}^{2} ; k_{\beta}=1170 \mathrm{deg} / \mathrm{rad}^{2} .
$$

Thus, $h \nu_{z} / k=47^{\circ} ; h \nu_{\beta} / k=58^{\circ}$; and the root mean square amplitudes of motion are : $\delta z_{\mathrm{rms}}=0.15 \AA$ and $\delta \beta_{\text {rms }}=15^{\circ}$ at $80 \mathrm{~K}$. A plot of the minimum energy and the position of the minimum as a function of $\beta$ is shown in figure 3 ; clearly, the energy barrier is much too high to allow free tumbling of a $\mathrm{N}_{2}$ molecule on this surface at temperatures of interest (i.e., below $100 \mathrm{~K}$ ).

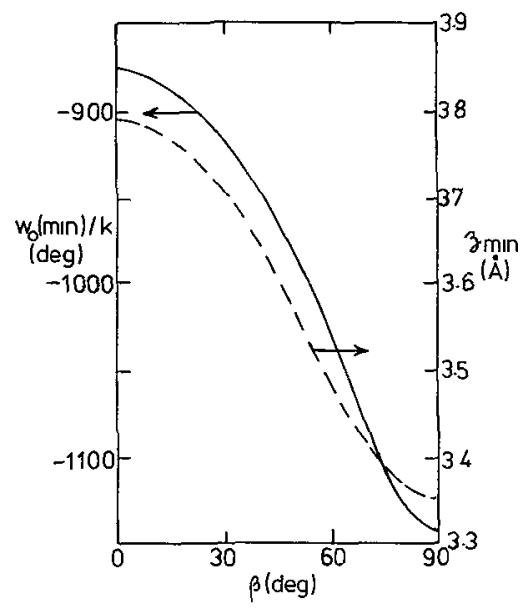

FIG. 3. - The minimum value of the gas-solid energy and the position of this minimum are plotted as a function of tilt angle $\beta$.

Of course, this argument implies that the variations in energy with position over the lattice and with azimuthal angle are not very large ; we now consider this part of $u_{s}(z, \tau, \beta, \alpha)$. For simplicity, we consider the case of $\beta=90^{\circ}$; eq. (2.1) then becomes

$$
u_{\mathrm{s}}\left(z^{*}, \boldsymbol{\tau}, \beta, \alpha\right)=2 w_{0}\left(z^{*}\right)+w_{1}\left(z^{*}\right) f(\tau, \alpha)
$$

$$
\text { where }
$$

$$
\begin{aligned}
f(\tau, \alpha)=-\sum_{n=1}^{3}\left\{\operatorname { c o s } \left[g \tau \sin \left(\gamma+\frac{2 \pi n}{3}\right)+\right.\right. \\
\left.+1.62 \sin \left(\alpha+\frac{2 \pi n}{3}\right)\right]+\cos \left[g \tau \sin \left(\gamma+\frac{2 \pi n}{3}\right)-\right. \\
\left.\left.\quad-1.62 \sin \left(\alpha+\frac{2 \pi n}{3}\right)\right]\right\}
\end{aligned}
$$

We evaluate these sums for three positions of the molecule : above the centre of a carbon hexagon (denoted by S) ; above a carbon atom (denoted by A) and above the mid-point between two carbon atoms (denoted by SP). After some trigonometric reduction, one finds :

$$
\begin{gathered}
f(\mathrm{~S}, \alpha)=2\{1-4 \cos [0.81 \sin \alpha] \times \\
\times \cos \left[0.81 \sin \left(\alpha+\frac{\pi}{3}\right)\right] \times \\
\left.\times \cos \left[0.81 \sin \left(\alpha-\frac{\pi}{3}\right)\right]\right\}, \\
f(\mathrm{AP}, \alpha)=-\frac{1}{2} f(\mathrm{~S}, \alpha) \\
\quad\{1+4 \cos [0.81 \sin \alpha] \times \\
\quad\left[0.81 \sin \left(\alpha+\frac{\pi}{3}\right)\right] \times \\
\left.\times \sin \left[0.81 \sin \left(\alpha-\frac{\pi}{3}\right)\right]\right\} .
\end{gathered}
$$

Plots of these functions are shown in figure 4 . The six-fold rotational symmetry of a homonuclear diatomic molecule centred at point $A$ or $S$ leads to very small variations in energy with azimuthal angle, whereas the two-fold symmetry at SP clearly gives much larger changes.

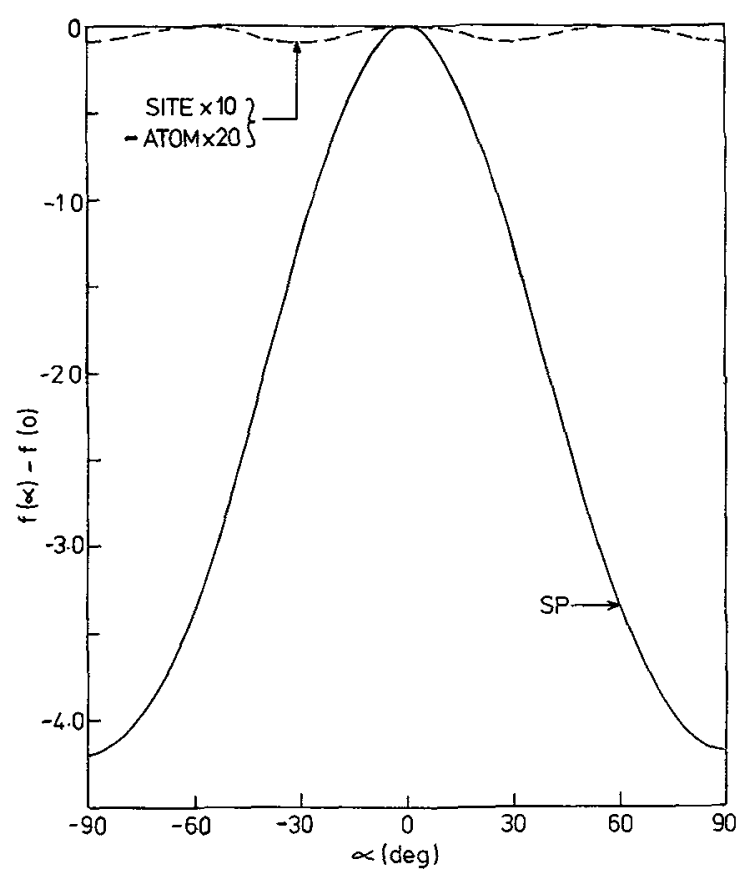

FIG. 4. - The weight function in the expression for the positional variation of the gas-solid energy for three $\mathrm{N}_{2}$-lattice positions. These are: centre-of-symmetry over the centre of a carbon hexagon (SITE) ; over a carbon atom (ATOM) ; and over the mid-point between two carbon atoms (SP). The weight function for a molecule lying flat on the surface is plotted as a function of the azimuthal angle $\alpha$ of the molecular symmetry axis which is defined in figure 1 . 
Assuming free rotation, one can average over $\alpha$ to give :

$$
\begin{aligned}
\langle f(\mathrm{~S}, \alpha)\rangle & =-6 J_{0}(1.62)=.-2.66 \\
\langle f(\mathrm{~A}, \alpha)\rangle & =1.33 \\
\langle f(\mathrm{SP}, \alpha)\rangle & =2 J_{0}(1.62)=0.88
\end{aligned}
$$

where $J_{0}$ is a zero order Bessel function. Note that these averages equal $-6,3$ and 2 in the limit of $L=0$ (an atom on the surface). Thus, the presence of a finite bond length has reduced the variations in energy over the surface by roughly a factor of two relative to the approximation of the molecule by an atom with $\sigma=3.36 \AA, \varepsilon / k=124^{\circ}$.

A plot of $w_{1}(z) / k$ for the $\mathrm{N}_{2}$-graphite system is shown in figure 5. For a value of $z$ near the minimum of energy, $w_{1}(z) / k=5 \mathrm{~K}$; if we denote the gas-solid energy averaged over $\alpha$ with $\beta=90^{\circ}$, $z=3.3 \AA$ by $\overline{u_{\mathrm{s}}(\tau)}$, we can now calculate that

$$
\begin{aligned}
\overline{u_{\mathrm{s}}(\mathrm{SITE}) / k} & =-1140-13(\mathrm{deg}) \\
\overline{u_{\mathrm{s}}(\mathrm{ATOM}) / k} & =-1140+7(\mathrm{deg}) \\
\overline{u_{\mathrm{s}}(\mathrm{SP}) / k} & =-1140+4(\mathrm{deg})
\end{aligned}
$$

for $z=3.3 \AA$.

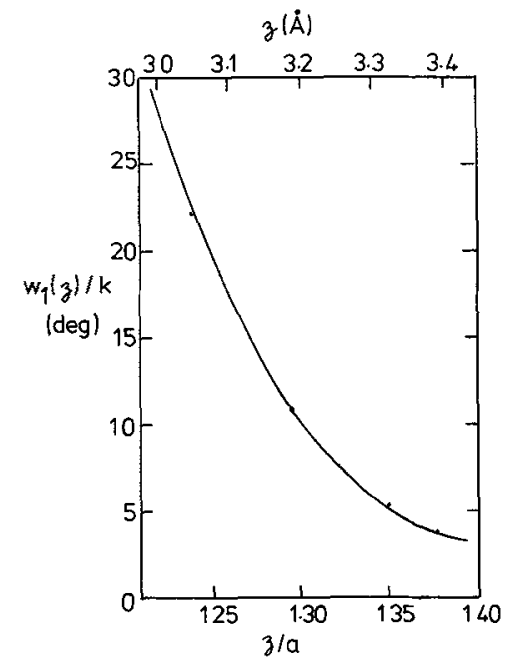

FIG. 5. - The distance dependence of the position-dependent part of the interaction is shown here. When multiplied by values of functions such as those in figure 4 , the variations in gas-solid energy with $\tau, \alpha, z$ are obtained (see eq. (2.7), for example).

Finally, the variation in energy at point SP as $\alpha$ changes from $0^{\circ}$ to $90^{\circ}$ with $\beta=90^{\circ}, z=3.3 \AA$ is $21 \mathrm{~K}$.

Before proceeding to a calculation of the pair-wise interactions between adsorbed $\mathrm{N}_{2}$ molecules, we should check the accuracy of this potential by comparison with experimental data, namely with the isosteric heat of adsorption in the limit of zero coverage. The best estimate of this quantity for $\mathrm{N}_{2}$-graphitised carbon black is [1]

$$
q_{\mathrm{st}}(0)=2190 \mathrm{cal} / \mathrm{mole} \text { at } 80 \mathrm{~K} \text {. }
$$

Assuming free azimuthal rotation and free translation parallel to the surface, and harmonic libration and translation perpendicular to the surface, one has

$$
\begin{aligned}
q_{\mathrm{st}} & =\frac{5}{2} R T-\left(u_{\min }+\frac{7}{2} R T\right) \\
& =2110 \mathrm{cal} / \mathrm{mole} .
\end{aligned}
$$

The agreement between theory and experiment could be improved slightly by adding the energy due to site preference; however, the difference is already smaller than the combined uncertainties in the two numbers.

3. $\mathrm{N}_{2}-\mathrm{N}_{2}$ Interactions. - If all the relevant coordinates are taken into account, the calculation of the interactions of pairs of adsorbed $\mathrm{N}_{2}$ molecules becomes extremely lengthy. However, one can still characterise the important features by neglecting the rather small energy variations associated with motion of the centre-of-mass of the pair parallel to the surface. This reduces the variables to $\tau_{12}, \beta_{1}, \beta_{2}, \alpha_{1}, \alpha_{2}$, if $z_{1}$ and $z_{2}$ are held fixed; in fact, the most probable configuration is $z_{1}=z_{2} \simeq z_{\operatorname{mix}} \simeq 3.3 \AA$. In this case the mutual interaction can still be computed from eq. (1.1)-(1.3), but it is necessary to redefine $X, Y, Z$ as : $X=\cos \beta$, $Y=\sin \beta \sin \alpha$ and $Z=\sin \beta \cos \alpha$.

For simplicity, the calculations presented here are also limited to the case where $\beta_{1}=\beta_{2}=\beta$. Figure 6

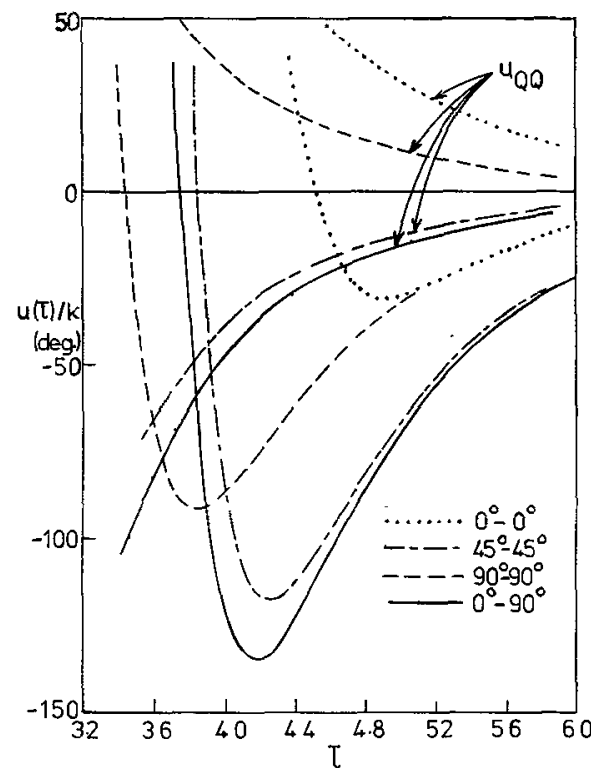

FIG. 6. - The mutual interaction of a pair of $\mathrm{N}_{2}$ molecules co-planar with each other and with a structureless surface. The quadrupolar part $u_{\mathrm{QQ}}$ and the total energy is plotted as a function of separation distance for various azimuthal orientations of the molecular symmetry axes relative to the centre-of-symmetry vector $\tau$. 
shows the interaction energy as a function of separation distance for a pair of $\mathrm{N}_{2}$ molecules lying flat on the surface $\left(\beta=90^{\circ}\right)$ for various azimuthal configurations. In addition, the quadrupolar part of the energy is shown. It is evident that this contribution is important for $\mathrm{N}_{2}$. For example, the T-shaped configuration is stabilised by quadrupolar energy ; in its absence, the parallel configuration with $\alpha_{1}=\alpha_{2}=90^{\circ}$ would be the most stable. As it is, the parallel configuration with $\alpha_{1}=\alpha_{2}=49.11^{\circ}$ is favourable for both quadrupolar energy and atomatom energies, and thus is nearly as stable as the T-shape. (Explicit expressions are :

$$
\begin{aligned}
\frac{u_{\mathrm{QQ}}}{k}= & \frac{30.3}{\tau^{* 5}}\left\{3-30 \cos ^{2} \alpha+35 \cos ^{4} \alpha\right\}, \text { (parallel) } \\
& =-\frac{121.2}{\tau^{* 5}},(\mathrm{~T}-\text { shape })
\end{aligned}
$$

with $\tau^{*}=\tau / 3.32 \AA$.)

The other important conclusion to be drawn from figure 6 is that the changes in energy that occur when a pair of co-planar $\mathrm{N}_{2}$ molecules rotate can be quite large. This means that care must be taken in performing statistical averages. The average mutual interaction of a pair of adsorbed molecules separated by a distance $\tau$ can be written :

$$
\bar{u}\left(\tau^{*}\right)=\frac{\int_{0}^{2 \pi} \int\left[u\left(\tau^{*}, \alpha_{1}, \alpha_{2}\right)\right]_{\beta} \exp \left(-\left[u\left(\tau^{*}, \alpha_{1}, \alpha_{2}\right)\right]_{\beta} / k T\right) \mathrm{d} \alpha_{1} \mathrm{~d} \alpha_{2}}{\int_{0}^{2 \pi} \int \exp \left(-\left[u\left(\tau^{*}, \alpha_{1}, \alpha_{2}\right)\right]_{\beta} / k T\right) \mathrm{d} \alpha_{1} \mathrm{~d} \alpha_{2}}
$$

where the notation []$_{\beta}$ means that some assumption must be made concerning the distribution of tilt angles $\beta_{1}, \beta_{2}$. Figure 7 shows averages calculated for some cases of interest : unweighted spherical average, where all values of $\beta_{1}, \beta_{2}, \alpha_{1}, \alpha_{2}$ are taken to be equally probable; and planar averages $\left(\beta_{1}=\beta_{2}=90^{\circ}\right)$, unweighted with respect to $\alpha_{1}, \alpha_{2}$, and then weighted according to eq. (3.3) with $T=80 \mathrm{~K}$. Since the variations in $\mathrm{N}_{2}$-solid energy with $\beta$ show that planar averages are the relevant ones at low temperature, the weighted average at $T=80 \mathrm{~K}$ should be a reasonably good representation of the true angle-averaged $\mathrm{N}_{2}-\mathrm{N}_{2}$ interaction at this temperature.

It is possible to make an approximate comparison of the theoretical $\mathrm{N}_{2}-\mathrm{N}_{2}$ potential with the experimental data for this system, because the general theory shows [11] that

$$
\lim _{N_{\mathrm{a}} \rightarrow 0}\left(\frac{\mathrm{d} q_{\mathrm{st}}}{\mathrm{d}\left(N_{\mathrm{a}} / \mathscr{A}\right)}\right)=\langle u(\tau)\rangle
$$

where $\mathscr{A}$ is the surface area. Although this slope is not accurately known in the limit of zero coverage, it is roughly constant across much of the submonolayer coverage regime (at $80 \mathrm{~K}$ ) [1]. In reduced units, one has

$$
\begin{aligned}
\mathscr{S}=\frac{\mathrm{d} q_{\mathrm{st}}}{\mathrm{d}\left(N_{\mathrm{a}} \sigma^{2} / \mathscr{A}\right)}=900 & \pm 100 \mathrm{cal} / \mathrm{mole} \\
& =\int_{0}^{\infty} u\left(\tau^{*}\right) p\left(\tau^{*}\right) \mathrm{d} \tau^{*},
\end{aligned}
$$

where $p\left(\tau^{*}\right)$ is the probability of finding a (spherical) atom at reduced distance $\tau^{*}$ from the central atom. The evaluation of this probability for dense two-dimensional fluids is non-trivial, even for spherical adsorbates. For the (12-6) interaction law, an approximate evaluation of the integral in equation (3.5) gave [11]

$$
\mathscr{P}=4.0 \varepsilon_{\mathrm{L}}
$$

where $\varepsilon_{L J}$ is the well depth of the potential. A more recent (and more accurate) evaluation gives the constant in eq. (3.6) to be 5.0 [12], with an estimated uncertainty of $\pm 15 \%$. Suppose we now assume that the mutual interactions of adsorbed $\mathrm{N}_{2}$ at $80 \mathrm{~K}$ can be approximated by the angle-averaged potential such as the one shown in figure 7. The experimental slopes lead one to expect $\varepsilon_{L S} / k=90 \mathrm{~K}$, which is in reasonable agreement with the calculated value of $101 \mathrm{~K}$ for the angle-averaged energy of co-planar $\mathrm{N}_{2}$ molecules. (A complete calculation requires not only that one average over $\beta_{1}, \beta_{2}$, but also that the probability $p\left(\tau^{*}, \alpha_{1}, \alpha_{2}, \beta_{1}, \beta_{2}\right)$ be calculated for a dense layer ; although it should be possible to adapt successful solutions of the analogous threedimensional problem to deal with the ad-molecule case, actual numerical evaluation of this function will require a great deal more effort.)

4. Discussion. - The ultimate goal of the theory presented here is to allow one to make some qualitative and ultimately, quantitative, statements about the translational and rotational motions in $\mathrm{N}_{2}$ films adsorbed on the basal plane of graphite. The recent neutron diffraction and thermodynamic studies of this system indicate that ordered layers are formed in registry with the substrate, i.e., with triangular symmetry and a nearest neighbour spacing of $4.25 \AA$. At coverages near the monolayer, the registered phase is stable up to $T \simeq 85 \mathrm{~K}$; at lower coverages the phase disorders into a liquid-like film at $T>50 \mathrm{~K}$; and at higher coverage, the monolayer appears to collapse into a denser, triangular-packed layer with spacing $=4.04 \AA$. Although the stability of a particular phase is determined by its free 


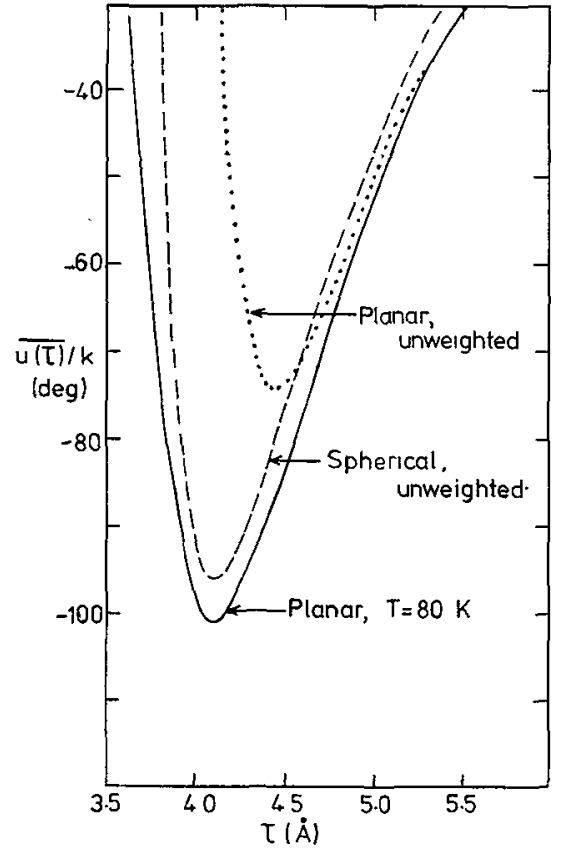

FIG. 7. - Average interaction energies of pairs of $\mathrm{N}_{2}$ molecules on a structureless surface. Two azimuthal averages are shown for co-planar molecules: for $T=\infty$ (unweighted) and for $T=80 \mathrm{~K}$; an unweighted average over tilt and azimuthal angle is also presented.

energy, the stable phases will generally be found to have configurations near the minimum in potential energy, especially if one allows for some kind of thermal motion. Thus the calculations reported here can be used in this connection and in particular can be helpful in deducing what kind of rotational motion exists in these films.

In the first place, we note that the difference in gas-solid energy between a registered and an unregistered film is calculated to be only $13 \mathrm{~K}$ per atom. Of course, this energy appears and disappears discontinuously as the lattice constant varies from less than to greater than $4.25 \AA$; nevertheless, it is clear that a minimum in the $\mathrm{N}_{2}-\mathrm{N}_{2}$ interaction at a separation near $4.25 \AA$ is also required if the registered phase is to be stable.

Secondly, the rather large barriers to free rotation for an isolated pair of $\mathrm{N}_{2}$ molecules co-planar with the surface will be materially reduced in the dense phase where each $\mathrm{N}_{2}$ has six neighbours in a regular array. Thus, it-seems likely that rotation in-plane will begin at a relatively low temperature ; heat capacity data seem to indicate $30-40 \mathrm{~K}$ [3].

If the molecules in the dense array are rotating freely, one can easily calculate the distances of minimum energy for fixed tilt angles ; for example, figure 4 indicates that $\tau_{\min }=4.45 \AA$ for co-planar imolecules (the unweighted average is appropriate in this case). Although this spacing is too large to allow the formation of a registered phase, we have also estimated a root mean square tilt angle of $75^{\circ}$ for an isolated molecule at $T=80 \mathrm{~K}$. To see the effect of deviations from co-planarity, the interaction of freely rotating molecules was calculated for various tilt angles $\beta=\beta_{1}=\beta_{2}$. Curves of these energies are plotted in figure 8 ; since these calculations are supposed to be relevant to a dense phase, the interactions with second and third neighbours in a hexagonal lattice were also included. As the tilt angle moves away from $90^{\circ}$, the net energy change per molecule in a hexagonal array should be calculated as the sum of the change in gas-solid energy plus three times the change in the average mutual interaction. Thus, for $\beta_{1}=\beta_{2}=60^{\circ}$, we estimate that the gas-solid energy increases by $110^{\circ}$ but the minimum mutual interaction decreases by $42^{\circ}$, relative to the co-planar configuration. Note that the distance of minimum energy is $4.25 \AA$ for this tilt angle.

Thus, it may be concluded that the registered phase at very low temperature consists of molecules lying flat on the surface with fixed azimuthal angles. As the temperature is raised, finite tilt angles are thermally excited and free azimuthal rotation begins (with the phase still in registry with the graphite basal plane). Furthermore, at $80 \mathrm{~K}$, the observed dense non-registered phase is probably made up of molecules undergoing free or nearly free rotation ; note that the minimum in the spherically averaged interaction of a pair of $\mathrm{N}_{2}$ molecules occurs at $\tau \simeq 4.10 \AA$ according to the curve in figure 7 , which is quite close to the value found for the dense unregistered phase.

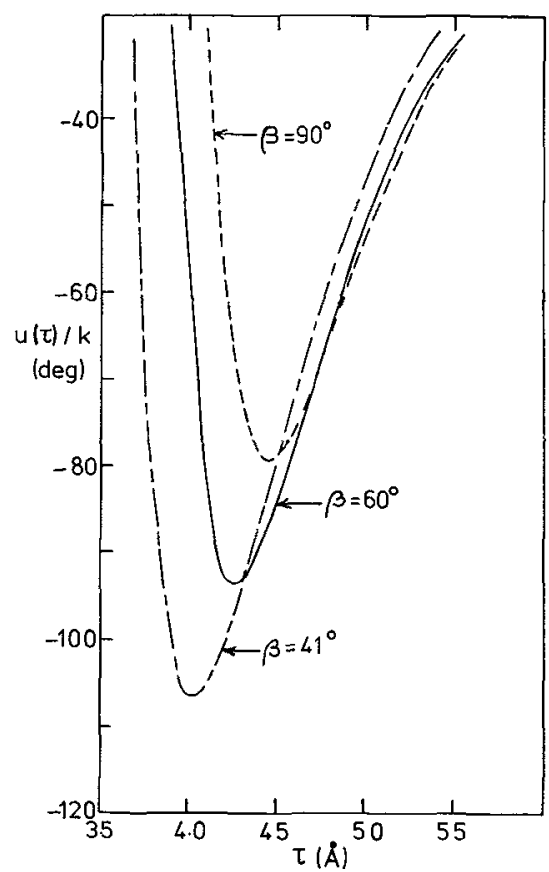

F1G. 8. - Azimuthal averages of the interaction of pairs of $\mathrm{N}_{2}$ molecules in a hexagonal array are shown for various values of the tilt angle $\beta$. Second and third neighbour interactions are added on, and it was assumed that changes in azimuthal angle produced negligible changes in energy when each molecule is symmetrically surrounded by six neighbours. 
The potential energy functions proposed in this paper give a good account of both the $\mathrm{N}_{2}$-solid and the $\mathrm{N}_{2}-\mathrm{N}_{2}$ interaction on the surface, as far as one can tell by comparison with the experimental heats of adsorption and with the general features of the phase diagram. Of course, a reasonably realistic model for the intermolecular interaction is a pre-requisite for detailed statistical mechanical theories of adsorbed films made up of non-spherical molecules. Until quite recently, the experimental characterisation of the $\mathrm{N}_{2}$-graphite system was not sufficiently detailed to make such efforts worthwhile. However, work reported and still in progress on this and similar examples (such as $\mathrm{O}_{2}$ on graphite [13] and NO on lamellar chalcogenides ( $\left.{ }^{1}\right)$ ) are yielding suitable data for theoretical analysis. It should be emphasised that an important consequence of non-sphericity in the molecular shape is a strong coupling between tilt angle and adsorbate-solid separation distance. It is therefore unlikely that the strictly two-dimensional statistical mechanical theories $[12,14]$ that have been successful in describing spherically symmetric adsorbates will be adequate for non-spherical molecules, except perhaps in the limits of low and high temperature.

\section{References}

[1] For a review of work on the interactions of various molecules (including $\mathrm{N}_{2}$ ) with graphitised carbon black, see.

Avgul, N. N. and Kiselev, A. V. Chemistry and Physics of Carbon, Vol. VI, ed. P. Walker Jr. (M. Dekker, New York), 1970)

[2] KJems, J. K., Passell, L., Taub, H., Dash, J. G. and Novaco, A. D., Phys. Rev. B 13 (1976) 1446.

[3] Rouquerol, J., PArtykA, S, and Rouquerol, F., JCS Faraday I 73 (1977) 306 ; ButLER, D. M., HuFF, G. B., Toth, R. W. and Stewart, G. A., Phys. Rev. Lett. 35 (1975) 1718 ; Chung, T. T. and DAsH, J. G., J. Chem Phys. 64 (1976) 1855 ; Surf. Sci., in press.

[4] MacRury, T. B., STeEle, W. A. and Berne, B. J., J. Chem. Phys. 64 (1976) 1288 ; Das Gupta, A., SANDler, S. I. and Steele, W. A., J. Chem. Phys. 62 (1975) 1769.

[5] Cheung, P. S. Y. and Powles, J. G., Mol. Phys. 32 (1976) 1383.

[6] English, C. A. and Venables, J. A., Proc. R. Soc. A 340 (1974) 57 ; Romano, S., Mol. Phys., in press ; RaICH, J. C. and Mills, R. L., J. Chem. Phys. 55 (1971) 1811 ; Goldman, V. V. and Klein, M., J. Chem. Phys. 64
(1976) 5121 ; RaICH, J. C., Gillis, N. S. and Koehler, T. R., J. Chem. Phys. 61 (1974) 1411 ; ZUnGER, A. and Huler, E., J. Chem. Phys. 62 (1975) 3016 ; SCHNEPP, O. and Ron, A., Discuss. Faraday Soc, 48 (1969) 26, 162.

[7] Stogryn, D. E. and Stogryn, A. P., Mol. Phys. 11 (1966) 371.

[8] STEeLE, W. A., The Interactions of Gases with Solid Surfaces (Pergamon, London, 1974), sections 2.8 and 4.9 ; Giamello, E., Pisani, C., Ricca, F. and Roetri, C., Surf. Sci. 49 (1975) 401 ; Derderian, E. J. and Steele, W. A., J. Chem. Phys., in press.

[9] Chen, C. H., SiskA, P. E. and LeE, Y. T., J. Chem. Phys. 59 (1973) 601 ; NG, C. Y., LEE, Y. T. and BARKER, J. A., J. Chem. Phys. 61 (1974) 1996.

[10] Steele, W. A., Surf. Sci. 36 (1973) 317.

[11] Réf. [8], Chap. 4.

[12] Steele, W. A., J. Chem. Phys. 65 (1976) 5256.

[13] Dericbourg, J., Surf. Sci. 59 (1976) 554 ; McTague, J. P. and NEILSEN, M., preprint.

[14] Henderson, D., J. Chem. Phys., in press.

(1) Y. Larher, private communication.

\section{DISCUSSION}

J. ROUQUEROL. - I would like to point out that, if the interaction energy of nitrogen with graphite which you find $\left(2110 \mathrm{cal} . \mathrm{mol}^{-1}\right)$ is somewhat lower than the one we obtain from actual calorimetric measurements $\left(2320 \mathrm{cal}^{\mathrm{mol}}{ }^{-1}\right)$, this may be explained by the residual heterogeneity of the actual sample which seems to affect the initial part of the calorimetric curve (cf. Fig. 1 in our paper). With a perfect graphite surface, we would expect a lower initial differential adsorption enthalpy, such as that obtained by extrapolating down to zero coverage the straight rising part of the calorimetric curve. 